\title{
Association of rs17095355 Polymorphism and Extrahepatic Biliary Atresia among Filipinos
}

\author{
Fresthel Monica M. Climacosa, ${ }^{1}$ Germana Emerita V. Gregorio ${ }^{2}$ and Catherine Lynn T. Silao ${ }^{2,3}$ \\ ${ }^{1}$ College of Medicine, University of the Philippines Manila \\ ${ }^{2}$ Department of Pediatrics, College of Medicine and Philippine General Hospital, University of the Philippines Manila \\ ${ }^{3}$ Institute of Human Genetics, National Institutes of Health, University of the Philippines Manila
}

\begin{abstract}
Background. Extrahepatic biliary atresia (EHBA) causes a rare obstructive cholestasis in infants. Kasai portoenterostomy if done before the third month of life may relieve obstruction. Genetic predisposition has been implicated in EHBA etiopathogenesis with rs17095355 polymorphism having the strongest association. We determined the association between rs17095355 and EHBA susceptibility of Filipino children, and described the outcome in each genotype among timely operated patients.
\end{abstract}

Methods. Thirty-four histologically diagnosed EHBA patients and thirty-three age- and sex-matched controls were recruited. Genomic DNA was extracted from peripheral blood and subjected to PCR and direct sequencing. Success of surgery among patients operated before 90 days of life was assessed by jaundice clearance six months post-surgery and native liver survival two and five years post-surgery.

Results. There was no significant difference among individuals carrying $\mathrm{T}$ and $\mathrm{C}$ alleles in developing EHBA (OR:1.36; $95 \% \mathrm{Cl}: 0.65-2.86)$. Jaundice persisted post-operatively in $75 \%$, $33 \%$ and $27 \%$ of Kasai-operated homozygous $\mathrm{T}(\mathrm{T} / \mathrm{T})$, homozygous $\mathrm{C}(\mathrm{C} / \mathrm{C})$ and heterozygous $(\mathrm{C} / \mathrm{T})$ patients, respectively. Fifty percent of Kasai-operated $C / C$ and $C / T$ patients retained their native liver whereas all Kasai-operated $\mathrm{T} / \mathrm{T}$ patients required liver transplantation two years post-surgery.

Conclusion. There is insufficient evidence to associate rs17095355 in EHBA development among Filipinos. Further investigation is warranted to elucidate genetic mechanisms in EHBA etiopathogenesis.

Key Words: biliary atresia, single nucleotide polymorphism, hepatic portoenterostomy

\section{Introduction}

Extrahepatic biliary atresia (EHBA) is an infantile disorder characterized by progressive inflammatory

Corresponding author: Fresthel Monica M. Climacosa

College of Medicine

University of the Philippines Manila

547 Pedro Gil St., Ermita, Manila 1000 Philippines

Telephone: +639434744737

Email: fres.climacosa@gmail.com obliteration and obstruction of the biliary systems resulting in disruption of bile flow ultimately leading to cirrhosis and liver failure. EHBA is considered the most serious liver disease affecting infants and is the most prevalent and important neonatal hepatobiliary disorder. The incidence of EHBA ranges from 1 in 5,000 to 1 in 19,000 live births in different populations, with the highest incidence occurring in Asia and the Pacific region. ${ }^{1}$ In the Philippine General Hospital (PGH), 10 to 15 new cases are diagnosed yearly.

Currently, the only available treatment for EHBA is Kasai portoenterostomy. However, $65 \%$ to $80 \%$ of operated patients on or before their third month of life still develop cirrhosis and progressive fibrosis. ${ }^{2}$ Liver transplantation is then consequently needed. EHBA represents the most frequent indication for pediatric liver transplantation worldwide. Different prognostic factors such as the patient's age at operation, histological features at diagnosis and duct remnant length at porta hepatis are predictors for the clinical outcome. Researches linking genetic factors and the outcome of Kasai operation in EHBA patients have not been conducted.

The etiopathogenesis of EHBA remains unclear. Recent studies implicated genetic predisposition and immunologic dysfunction as potential causes of EHBA. Polymorphisms in genes with vital roles in initiation and propagation of various inflammatory responses (e.g. cluster of differentiation $14,{ }^{3}$ macrophage migration inhibitory factor, ${ }^{4}$ intercellular adhesion molecule-1,5 vascular endothelial growth factor ${ }^{6}$ and tumor necrosis-alpha ${ }^{7}$ ) were investigated for possible links to EHBA susceptibility. Transcriptome profiling likewise suggest deregulated gene expression related to immunity and inflammatory responses. $^{8} \mathrm{~A}$ genome-wide association study (GWAS) performed by Garcia-Barcelo et al in 2010 identified the putative EHBA susceptibility locus in band 24 on the long arm of chromosome $10 \quad(10 \mathrm{q} 24) .{ }^{9} \quad$ The single nucleotide polymorphism (SNP) with the strongest overall association to EHBA was found for rs17095355 on this locus. Association studies between gene polymorphisms and development of EHBA in Filipino infants have not yet been explored.

This case-control study aimed to determine the association between the rs17095355 SNP and the 
susceptibility of Filipino infants in developing EHBA and to describe the outcome of patients who underwent Kasai portoenterostomy before their third month of life in each genotype.

\section{Methods}

\section{Study Participants}

The study protocol was approved by the Research and Ethics Board of the College of Medicine, University of the Philippines Manila. All study participants, parents, legal guardians were fully informed regarding the study protocol and its procedures prior to participating in the study. Written informed consents were obtained from the parents and/or legal guardians of all study participants and oral assents from patients aged 8 to 10 years old were obtained.

\section{$\underline{\text { Cases }}$}

Thirty-four Filipino children histologically diagnosed to have perinatal EHBA between January 2000 and December 2010 at the Section of Pediatric Gastroenterology Hepatology and Nutrition of the University of the Philippines Philippine General Hospital were recruited. The patients were diagnosed with the disease between 0 to 12 months of age but were recruited only at the time of the study. None of these patients had associated congenital anomalies.

Patients who have undergone Kasai portoenterostomy before three months of life between January 2000 and December 2005 were identified. Successful operation was based on the disappearance of jaundice (total bilirubin $<20$ $\mu \mathrm{mol} / \mathrm{L})$ after six months of the operation. The survival of the patient's native liver 2 and 5 years after the surgery was also assessed. In all patients who had a Kasai portoenterostomy operation, the surgery was done by experienced pediatric surgeons of the institution.

\section{Controls}

Thirty-three age- and sex-matched patients with no known liver disease were recruited as controls from the Outpatient Department (OPD) of the Philippine General Hospital between April 2011 and February 2012.

\section{Polymorphism Analysis}

Genomic DNA was extracted from $4 \mathrm{~mL}$ peripheral blood of all study participants (cases and controls) using the QIAGEN DNA Midi Kit (QIAGEN, Valencia, CA, USA).

The 340-bp region containing rs17095355 was amplified using the forward primer (5'-AAG AAT GGG GAA GAA CAA GT- $3^{\prime}$ ) and the reverse primer (5'-TCA GTA ATT CCA GGG GCT A-3'). The reaction mixture was composed of $100 \mathrm{ng}$ DNA template, $0.5 \mathrm{mM}$ of each primer, 1.25X PCR buffer, $1.5 \mathrm{mM} \mathrm{MgCl}$, $0.2 \mathrm{mM} \mathrm{dNTPs,} \mathrm{PCR-}$ grade water and 2.5 units of DNA polymerase. PCR was carried out in an ABI Thermal Cycler using the following thermal cycling conditions: initial denaturation at $94^{\circ} \mathrm{C}$ for 5 min, 35 amplification cycles each consisting of denaturation at $94^{\circ} \mathrm{C}$ for $1 \mathrm{~min}$, annealing at $58^{\circ} \mathrm{C}$ for $1 \mathrm{~min}$ and extension at $72^{\circ} \mathrm{C}$ for $1 \mathrm{~min}$, and a final extension at $72^{\circ} \mathrm{C}$ for $10 \mathrm{~min}$.

PCR products were analyzed by electrophoresis on $2 \%$ agarose gels stained with $0.5 x$ GelRed Nucleic Acid Stain (Biotium Inc., Hayward, CA, USA). Purified PCR products were subsequently sequenced using the ABI capillary system (Macrogen Research, Seoul, Korea). Sequences were compared using BLAST (http://www.ncbi.nlm.nih.gov/BLAST/).

\section{Statistical Analysis}

Student's t-test was employed to compare the demographic characteristics of both cases and controls. Allelic and genotypic frequencies were compared through the $x^{2}$ test. Using $2 \times 2$ or $2 \times 3$ contingency tables, odds ratio together with $95 \%$ confidence interval were computed. All statistical analysis was performed using Epi Info ${ }^{\text {TM }}$ 3.5.1. A p-value of less than 0.05 was considered statistically significant.

\section{Results}

Sixty-seven participants, 34 EHBA cases and 33 controls, were recruited in the study. Mean age of case and controls were 4.9 and 5.0 years, respectively, with male-tofemale ratio of approximately 1:2 (Table 1 ). Among the EHBA patients recruited, $25(73.5 \%)$ of them underwent Kasai portoenterostomy before their third month of life.

Table 1. Demographic characteristics of the study groups

\begin{tabular}{lccc}
\hline & EHBA $(n=34)$ & Control $(n=33)$ & p-value \\
\hline Age in years (mean \pm SD) & $4.90 \pm 3.21$ & $5.04 \pm 3.11$ & 0.85 \\
Sex (Male:Female) & $22: 12$ & $24: 9$ & 0.50 \\
\hline
\end{tabular}

Allelic frequencies for $\mathrm{C}$ and $\mathrm{T}$ alleles for the control group were 0.59 and 0.41 , respectively, and 0.51 and 0.49 for EHBA group. There was no significant difference among individuals carrying the $\mathrm{T}$ allele (OR: 1.36 ; 95\% CI: 0.65 to 2.86) and those carrying the $C$ allele in the likelihood of developing EHBA (Table 2).

Table 2. Distribution of rs17095355 allele and genotype among extrahepatic biliary atresia patients and control group

\begin{tabular}{ccccc}
\hline \multirow{2}{*}{$\begin{array}{c}\text { rs17095355 } \\
\text { polymorphism }\end{array}$} & $\begin{array}{c}\text { EHBA } \\
(\mathrm{n}=34)\end{array}$ & $\begin{array}{c}\text { Control } \\
(\mathrm{n}=33)\end{array}$ & $\begin{array}{c}\text { Odds Ratio } \\
(95 \% \mathrm{CI})\end{array}$ & p-value \\
\hline Genotype & & & & \\
$\mathrm{C} / \mathrm{C}$ & $7(20.59)$ & $9(27.27)$ & & \\
$\mathrm{C} / \mathrm{T}$ & $21(61.76)$ & $21(63.64)$ & & \\
$\mathrm{T} / \mathrm{T}$ & $6(17.65)$ & $3(9.09)$ & $2.14(0.41$ to 14.35$)$ & 0.25 \\
\hline Allele & $35(51.47)$ & $39(59.09)$ & & \\
$\mathrm{C}$ & $33(48.53)$ & $27(40.91)$ & $1.36(0.65$ to 2.86$)$ & 0.38 \\
\hline $\mathrm{T}$ & & & &
\end{tabular}


Of the 25 EHBA cases who had portoenterostomy operation before three months, genotypic distribution according to jaundice clearance within six months after operation showed that three of four (75\%) Kasai-operated patients with $\mathrm{T} / \mathrm{T}$ genotype had persistent jaundice as compared to two of six (33\%) with C/C and four of 15 with $\mathrm{C} / \mathrm{T}(26.67 \%)$ genotypes (Table 3$)$. The remaining nine EHBA patients did not undergo Kasai due to late diagnosis (i.e. after 90 days of life) but were advised liver transplantation. Six of them underwent liver transplantation while the remaining three died from the complications of EHBA. Their genotypes are as follows: $1 \mathrm{C} / \mathrm{C}, 6 \mathrm{C} / \mathrm{T}$ and $2 \mathrm{~T} / \mathrm{T}$.

Table 3. Distribution of Kasai-operated extrahepatic biliary atresia patients according to rs17095355 genotype and outcome of operation after 6 months

\begin{tabular}{ccc}
\hline \multirow{2}{*}{ rs17095355 genotype } & \multicolumn{2}{c}{ Kasai-operated EHBA patient $(\mathrm{n}=25), \mathrm{n}(\%)$} \\
\cline { 2 - 3 } & Jaundice Clearance & Persistent Jaundice \\
\hline $\mathrm{C} / \mathrm{C}$ & $4(66.67)$ & $2(33.33)$ \\
$\mathrm{C} / \mathrm{T}$ & $11(73.33)$ & $4(26.67)$ \\
$\mathrm{T} / \mathrm{T}$ & $1(25)$ & $3(75)$ \\
\hline
\end{tabular}

With regards the condition of the native liver after Kasai portoenterostomy, all operated EHBA cases with the T/T genotype required liver transplantation two years after surgery while only $53 \%$ and $50 \%$ of $\mathrm{C} / \mathrm{T}$ and C/C EHBA patients, respectively, needed transplantation (Table 4). This proportion of EHBA cases requiring liver transplantation after Kasai increases $(60 \%)$ among $\mathrm{C} / \mathrm{T}$ while unchanged (still $50 \%$ ) among C/C EHBA patients five years after surgery.

Table 4. Distribution of Kasai-operated extrahepatic biliary atresia patients according to rs17095355 genotype and condition of native liver after 2 and 5 years the surgery

\begin{tabular}{ccccc}
\hline \multirow{2}{*}{ rs17095355 genotype } & \multicolumn{3}{c}{ Kasai-operated EHBA patient $(\mathrm{n}=25), \mathrm{n}(\%)$} \\
\cline { 2 - 5 } & \multicolumn{2}{c}{ Native liver } & \multicolumn{2}{c}{ Liver transplant } \\
\cline { 2 - 5 } & After 2yrs & After 5yrs & After 2yrs & After 5yrs \\
\hline $\mathrm{C} / \mathrm{C}$ & $3(50)$ & $3(50)$ & $3(50)$ & $3(50)$ \\
$\mathrm{C} / \mathrm{T}$ & $7(46.67)$ & $6(40)$ & $8(53.33)$ & $9(60)$ \\
$\mathrm{T} / \mathrm{T}$ & $0(0)$ & $0(0)$ & $4(100)$ & $4(100)$ \\
\hline
\end{tabular}

\section{Discussion}

\section{Rs17095355 polymorphism and EHBA development}

The rs17095355 polymorphism located at band 24 on the long arm of chromosome 10 was identified as the SNP most associated with the development of EHBA in a GWAS. ${ }^{9}$ This association was verified among Chinese, ${ }^{10-11}$ Thai $^{12}$ and Caucasian $^{13}$ populations. A recent meta-analysis of these studies reported that the $\mathrm{T}$ allele predisposes an individual in developing EHBA with a pooled OR of 1.72. ${ }^{14}$ Rs17095355 is positioned between two genes, ADD3 and XPNPEP1, which encode substances relevant to liver functions.

$A D D 3$ encodes for adducin 3, a membrane skeletal protein that has been shown to play major roles in the assembly of the spectrin-actin network of cell membranes. ${ }^{15}$ Bile flow is controlled by the bile canalicular membrane-associated filaments (BCMF), particularly actin and myosin. Impairment of these interactions was demonstrated to cause severe cholestasis. ${ }^{16}$ In fact, BCMF depositions around bile canaliculi have been reported in EHBA patients who did not exhibit bile flow after Kasai operation. ${ }^{17}$

On the other hand, XPNPEP1 encodes the soluble $\mathrm{X}$ prolyl aminopeptidase 1 that catalyzes the cleavage of the $\mathrm{N}$ terminal amino acid adjacent to a proline residue. ${ }^{18}$ It was reported that this aminopeptidase is expressed in both adult and fetal hepatobiliary epithelial cells and is responsible for the sequential degradation and regulation of the inflammatory mediators, bradykinin and substance P. ${ }^{19,20}$ Spivak et al in separate studies showed that substance $\mathrm{P}$ is able to change the level of bile secretion and chemical composition and affect various enzyme systems in the hepatocyte. ${ }^{21}$ Increased levels of SP in cholestasis, ${ }^{22}$ liver cirrhosis $^{23}$ and chronic liver disease ${ }^{24}$ were also reported.

These evidences substantiate the role of $A D D 3$ and/or XPNPEP1 in EHBA development, probably by disrupting the regulatory systems involved in inflammatory processes. Recently, however, it was demonstrated that knockdown of $A D D 3$ but not XPNPEP1 gene led to intrahepatic defects and decreased biliary function in zebrafish. ${ }^{25}$

In this study, however, association between rs17095355 polymorphism and EHBA among Filipinos was not demonstrated. True association between the SNP and disease may not have been observed due to the small sample size, which could affect the statistical power of the study. ${ }^{26}$ In contrast to Chinese and Thai EHBA patients, the lack of association of EHBA to rs17095355 SNP in Filipino children in this study could also be attributed to genetic differences contributed by ethnicity. Several meta-analyses reported that particular SNPs may be correlated to a disease in a specific population but not in others as in chronic hepatitis B infections ${ }^{27}$ and drug-induced hepatic toxicity. ${ }^{28}$ Further investigations are needed to identify genetic factors in EHBA development among Filipinos.

\section{Rs17095355 polymorphism and outcome of Kasai operation}

Current protocols in the surgical management of EHBA indicate performing the operation on or before the third month of patient's life. However, about $60 \%$ of operated patients still developed severe complications like cholangitis, portal hypertension and gastrointestinal bleeding which would eventually require them to undergo liver transplantation. ${ }^{29}$

Many parameters were suggested as prognosticating factors in the success of hepatic portoenterostomy. This includes age at Kasai, ${ }^{30}$ levels of liver function tests (aspartate and alanine aminotransferase; total, direct and indirect bilirubin), ${ }^{31}$ presence of associated anomaly, ${ }^{32}$ bile duct remnants at the porta hepatis, ${ }^{33}$ signs of fibrosis and/or 
cirrhosis $^{34}$ in histologic analysis as well as the experience of the surgeon and the center. ${ }^{35}$

To our knowledge, this study is the first to describe the outcome of Kasai according to a genetic factor. All patients who had Kasai portoenterostomy were operated before three months and the surgery was done only in one institution. Although other prognostic factors were not controlled, the results of this study suggest that individuals carrying the $\mathrm{T}$ allele may have poorer outcome after surgery even when the procedure was done before their third month of life. Recent evidence indicates that perioperative risks and outcomes may be significantly influenced by the individual's genotype. ${ }^{36}$ After controlling possible confounding factors, surgical patients carrying the G-572C allele and in patients homozygous for the G-174C allele in the interleukin-6 (IL-6) gene were found to have elevated pro-inflammatory cytokines after cardiopulmonary bypass. ${ }^{37}$ Site specific mutations in the coding region of the tumor necrosis factoralpha (TNF- $\alpha$ ) gene were associated with increased levels of TNF- $\alpha .{ }^{38}$ These evidences point to increased risk of adverse perioperative outcomes due to allotypic variation leading to perturbations in the pro-inflammatory and antiinflammatory cytokine balance. ${ }^{36}$

\section{Conclusion}

Our study showed no significant association between rs17095355 and EHBA development in Filipino children. Further investigation is warranted to ascertain genetic mechanism in EHBA etiopathogenesis.

\section{Acknowledgments}

We extend our gratitude to the Institute of Human Genetics of the National Institutes of Health and the MD-PhD committee of the University of the Philippines College of Medicine for their insights and suggestions in conducting this study.

Statement of Authorship

All authors have approved the final version submitted.

\section{Author Disclosure}

All authors declared no conflict of interest.

\section{Funding Source}

This paper was funded by the Philippine Council for Health Research and Development of the Department of Science and Technology.

\section{References}

1. Chardot C. Biliary atresia. Orphanet J Rare Dis. 2006; 1:28.

2. Otte JB, de Ville de Goyet J, Reding R, et al. Sequential treatment of biliary atresia with Kasai portoenterostomy and liver transplantation: A review. Hepatology. 1994; 20(1 Pt 2):41S-48S.
3. Shih $\mathrm{HH}$, Lin TM, Chuang JH, et al. Promoter polymorphism of the CD14 endotoxin receptor gene is associated with biliary atresia and idiopathic neonatal cholestasis. Pediatrics. 2005; 116(2):437-41.

4. Arikan C, Berdeli A, Ozgenc F, Tumgor G, Yagci RV, Aydogdu S. Positive association of macrophage migration inhibitory factor gene 173G/C polymorphism with biliary atresia. J Pediatr Gastroenterol Nutr. 2006; 42(1):77-82.

5. Arikan C, Berdeli A, Kilic M, Tumgor G, Yagci RV, Aydogdu S. Polymorphisms of the ICAM-1 gene are associated with biliary atresia. Dig Dis Sci. 2008; 53(7):2000-4.

6. Lee HC, Chang TY, Yeung CY, et al. Genetic variation in the vascular endothelial growth factor gene is associated with biliary atresia. J Clin Gastroenterol. 2010; 44(2):135-9.

7. Fotouhi N, Bonyadi M, Jahanafrooz Z, et al. Tumour necrosis factoralpha gene polymorphisms in Iranian patients with biliary atresia. Afr J Paediatr Surg. 2014; 11(3):233-7.

8. Xiao J, Xia SY, Xia Y, Xia Q, Wang XR. Transcriptome profiling of biliary atresia from new born infants by deep sequencing. Mol Biol Rep. 2014; 41(12):8063-9.

9. Garcia-Barcelo MM, Yeung MY, Miao XP, et al. Genome-wide association study identifies a susceptibility locus for biliary atresia on 10q24.2. Hum Mol Genet. 2010; 19(14):2917-25.

10. Cheng G, Tang CS, Wong EH, et al. Common genetic variants regulating ADD3 gene expression alter biliary atresia risk. J Hepatol. 2013; 59(6):1285-91

11. Zeng S, Sun $P$, Chen Z, et al. Association between single nucleotide polymorphisms in the ADD3 gene and susceptibility to biliary atresia. PLoS One. 2014; 9(10):e107977.

12. Kaewkiattiyot S, Honsawek S, Vejchapipat P, Chongsrisawat P, Poovorawan V. Association of X-prolyl aminopeptidase 1 rs17095355 polymorphism with biliary atresia in Thai children. Hepatol Res. 2011; 41(12):1249-52.

13. Tsai EA, Grochowski CM, Loomes KM, et al. Replication of a GWAS signal in a Caucasian population implicates ADD3 in susceptibility to biliary atresia. Hum Genet. 2014; 133(2):235-43.

14. Li J, Gao W, Zuo W, Liu X. Association between rs17095355 polymorphism on 10q24 and susceptibility to biliary atresia: a metaanalysis. J Matern Fetal Neonatal Med. 2017; 30(15):1882-6.

15. Citterio L, Tizzoni L, Catalano M, Zerbini G, Bianchi G, Barlassina C. Expression analysis of the human adducin gene family and evidence of ADD2 beta4 multiple splicing variants. Biochem Biophys Res Commun. 2003; 309(2):359-67.

16. Oshio C, Phillips MJ. Contractility of bile canaliculi: Implications for liver function. Science. 1981; 212(4498):1041-2.

17. Segawa O, Miyano T, Fujimoto T, Watanabe S, Hirose M, Fujiwara T. Actin and myosin deposition around bile canaliculi: a predictor of clinical outcome in biliary atresia. J Pediatr Surg. 1993; 28(6):851-6.

18. LiX, Lou Z, Li X, et al. Structure of human cytosolic X-prolyl aminopeptidase: A double $\mathrm{MN}(\mathrm{II})$-dependent dimeric enzyme with a novel three-domain subunit. J Bio Chem, 2008; 283(33):22858-66.

19. Vanhoof G, de Meester I, Hendriks D, et al. Proline-specific aminopeptidases: potential role in bradykinin degradation. Agents Actions Suppl. 1992; 38(Pt 2):120-7.

20. Kitamura S, Carbini LA, Simmons WH, Scicli AG. Effects of aminopeptidase $\mathrm{P}$ inhibition on kinin-mediated vasodepressor responses. Am J Physiol. 1999; 276(5 Pt 2):1664-71.

21. Spivak LS, Makarchuk Mlu, Liashchenko TP, Veselskyi SP. Effect of natural tachykinins on the bile production in rats. Fiziol Zh. 2005; 51(4):61-4.

22. Trivedi M, Bergasa NV. Serum concentrations of substance $P$ in cholestasis. Ann Hepatol. 2010; 9(2):177-80.

23. Lee FY, Lin HC, Tsai YT, et al. Plasma substance P levels in patients with liver cirrhosis: relationship to systemic and portal hemodynamics. Am J Gastroenterol. 1997;92(11):2080-4.

24. El-Raziky MS, Gohar N, El-Raziky M. Study of substance P, renin and aldosterone in chronic liver disease in Egyptian children. J Trop Pediatr. 2005; 51(5):320-3.

25. Tang V, Cofer ZC, Cui S, Sapp V, Loomes KM, Matthews RP. Loss of a candidate biliary atresia susceptibility gene, add3a, causes biliary 
developmental defects in zebrafish. J Pediatr Gastroenterol Nutr. 2016; 63(5):524-30.

26. Hong EP, Park JW. Sample size and statistical power calculation in genetic association studies. Genomics Inform. 2012; 10(2):117-22

27. Wei Y, Tian Q, Li L, Zhang D. Association between IFN- $\gamma$ genetic polymorphisms and susceptibility to hepatitis B virus infection: a metaanalysis. Ann Hum Biol. 2016; 43(6): 527-36.

28. Li C, Long J, Hu X, Zhou Y. GSTM1 and GSTT1 genetic polymorphisms and risk of anti-tuberculosis drug-induced hepatotoxicity: an updated meta-analysis. Eur J Clin Microbiol Infect Dis. 2013; 32(7):859-68.

29. Bijl EJ, Bharwani KD, Houwen RH, de Man RA. The long-term outcome of the Kasai operation in patients with biliary atresia: a systematic review. Neth J Med. 2013; 71(4):170-3.

30. Ohi R. Biliary atresia: A surgical perspective. Clin Liver Dis. 2000; 4(4):779-804.

31. Goda T, Kawahara H, Kubota A, et al. The most reliable early predictors of outcome in patients with biliary atresia after Kasai's operation. J Pediatr Surg. 2013; 48(12):2373-7.

32. Davenport M, Savage M, Mowat AP, Howard ER. Biliary atresia splenic malformation syndrome: An etiologic and prognostic subgroup. Surgery. 1993; 113(6):662-8.
33. Chandra RS, Altman RP. Ductal remnants in extrahepatic biliary atresia: A histopathologic study with clinical correlation. J Pediatr. 1978; 93(2):196-200.

34. Balistreri WF, Grand R, Hoofnagle JH, et al. Biliary atresia: Current concepts and research directions. Summary of a symposium. Hepatology. 1996; 23(6):1682-92.

35. Sokol RJ, Mack C, Narkewicz MR, Karrer FM. Pathogenesis and outcome of biliary atresia: Current concepts. J Pediatr Gastroenterol Nutr. 2003; 37(1):4-21.

36. Ziegler S, Tsusaki BE, Collard CD. Influence of genotype on perioperative risk and outcome. Anesthesiology. 2003; 99(1):212-9.

37. Brull DJ, Montgomery HE, Sanders J, et al. Interleukin-6 gene $-174 \mathrm{~g} \rightarrow \mathrm{c}$ and $-572 \mathrm{~g} \rightarrow \mathrm{c}$ promoter polymorphisms are strong predictors of plasma interleukin-6 levels after coronary artery bypass surgery. Arterioscler Thromb Vasc Biol. 2001; 21:1458-63.

38. Stuber F, Petersen M, Bokelmann F, Schade U. A genomic polymorphism within the tumor necrosis factor locus influences plasma tumor necrosis factor alpha concentrations and outcome of patients with severe sepsis. Crit Care Med. 1996; 24(3):381-4. 